\title{
LOW DIVERGENCE LASER-PLASMA-BASED BEAMS
}

\author{
N. Saleh, S. Chen, S-Y. Chen, A. Maksimchuk, D. Umstadter, X. Wang, P. Zhang \\ Center for Ultrafast Optical Science, MI 48109 , USA \\ R. Crowell \\ Argonne National Laboratory, Argonne, IL 60439, USA \\ K. Assamagan, P. Gueye, C. Keppel \\ Hampton University, VA 23668, USA
}

\begin{abstract}
We discuss the mechanisms, applications, and extraction of low-divergence laser-plasma-based electron beams that represent our past, current, and future research activities in the Center for Ultrafast Optical Science at the University of Michigan. We show experimentally that these beams have superior transverse emittance of $0.06 \mathrm{pi} \mathrm{mm} \bullet \mathrm{mrad}$, along with $n C$ charge, suitable for various applications, like pulse radiolysis. We also report progress in implementing a scheme to produce a mono-energetic electron beam [1], along with an electromagnetic QQQDD spectrometer system especially built for characterizing such beam.
\end{abstract}

\section{PRODUCTION}

Discussed below, are schemes to produce electron beams within our research program.

\subsection{Long Pulse regime}

In the long laser pulse regime, also known as SelfModulated Laser Wakefield Accelerator (SMLWFA), a long laser pulse, compared to the plasma period, excites a wakefield or a plasma wave via Raman Forward Scattering (RFS). The plasma wave grows with the laser pulse, during which, Raman Backwards Scattering (RBS) along with other mechanisms, like heating and possibly breaking, uncontrollably injects electrons into the plasma wave.

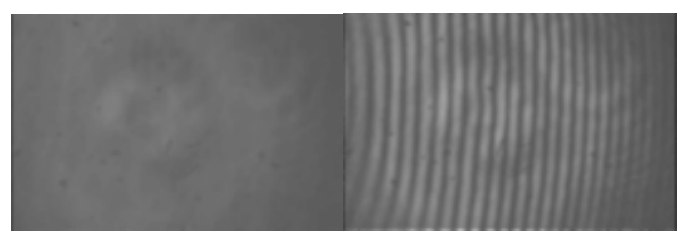

Fig 1: Interference is evidence for spatio-temporal overlap of pump and injection in air at low power (forward imaging). To the left is when the beam are not temporally overlapped, and to the right is when they are temporally overlapped.

Chen et al., [2] have discussed in details the dynamics of the SMLWA scheme, in addition to various interesting properties of the produced beam. We also performed a prototype experiment to investigate the scheme proposed in [1] but in the long laser pulse regime. In this experiment, two laser pulses, pump and injection, were crossed in a tenuous Helium plasma, we established evidence of spatio-temporal overlapped of the two laser fields both in air at low laser power, and in real experimental conditions at high laser power. Fig. 2 , constitutes the highest-power interference pattern ever recorded in a plasma, to our knowledge [3].
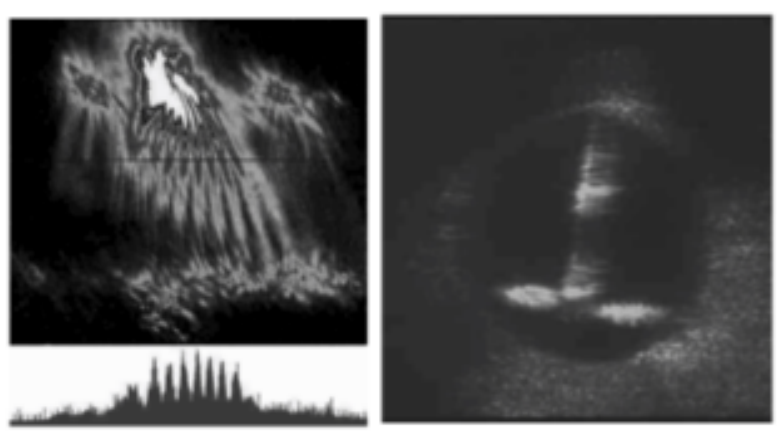

Fig 2: Interference (left) and extended propagation (right) due to change of refractive index are evidence for spatio-temporal overlap of pump and injection in vacuum at high power (side imaging from bisecting direction).

\subsection{Short Pulse Regime}

We have experimented in a new parameter regime with short laser pulses [4] (again compared to the plasma wave), where we scanned the plasma density, or equivalently the plasma wave period, in the vicinity of the resonant regime (when the laser pulse and the plasma wave period are equal). We observed interesting features in this regime that are also deemed detrimental for the resonant regime discussed next. We encountered the following: 1) the plasma wave exhibits not selftrapping in the vicinity of the resonant regime, this was manifested by the fact that no electron beam was detected above the noise level. 2) The laser beam tends to break up and filament when the laser power exceeds five times the critical power for self-focusing. 3) When the laser beam breaks up, an $\mathrm{MeV}$, low divergence (down to 1 degree) electron beam is produced in the forward direction. No Raman scattering was observed in this broad-bandwidth laser pulse regime. Thus, we believe that this electron beam is due to a new mechanism other than the SMLWFA. 
In the same short pulse regime, a new novel mechanism to produce low-divergence, monochromatic electron beam was proposed earlier [1]. This mechanism, the LILAC (Laser Injected Laser ACcelerator), utilizes two short laser pulses, pump and injection, overlapping in gaseous plasma. The former resonantly excites a 1-D wakefield, while the latter injects electrons in the correct phase of the former. Active investigation and implementation of this mechanism is ongoing. The feasibility of this mechanism seems promising based on the experimental results discussed above, see [5] for more details.
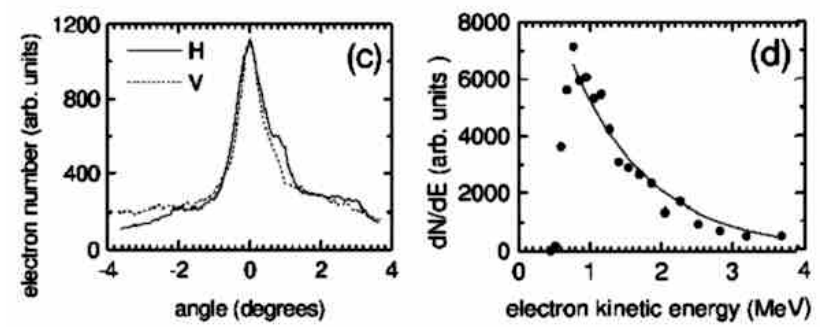

Fig 3: Electron beam divergence and temperature when a short laser pulse breaks up in plasma at $5 \mathrm{x}$ the critical power or more.

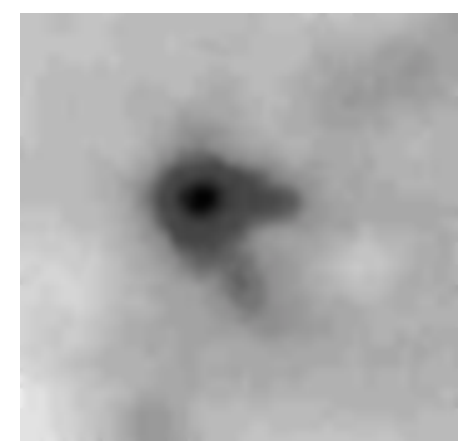

Fig 4: The electron beam transverse profile produced when a short laser pulse breaks up in plasma corresponding to the figure above.

\section{APPLICATIONS}

We have used the electron beam produced by the SMLWFA mechanism to perform an experiment in pulse Radiolysis [6]. By measuring the microsecond decay of the hydrated electrons produced by the electron beam ionization of liquid water, it has been shown that the electron pulses generated can provide sufficient charge to perform chemical studies. There are many advantages to using short pulse TW lasers for picosecond pulse radiolysis experiments. First, the technology already exists to generate $\mathrm{nC}$ electron pulses. Second, the cost of a TerraWatt laser systems is much less than the cost of a photo-cathode-driven linac. Third, for radiation chemists, the most important advantage is that the electron pulses are naturally synchronized with the laser pulses that generate them. This will allow the implementation of a wide variety of sensitive, laser-based detection methods that have been previously unavailable to the radiation chemists.

\section{EXTRACTION}

\subsection{Design}

We have designed a QQQDD electromagnetic spectrometer for extraction and analysis of the laserplasma based electron beams discussed above, this system is considered one of its kinds; there were reports on detectors for laser plasma electron beams in the literature [7], with little or no emphasis on extraction. This spectrometer, however, is intended to extract these beams form the production point to the delivery point where they can be used for various applications. The design of the optics in the beam line utilized the TRANSPORT beam transport code, further simulations were performed using PBO LAB by AccelSoft, Inc. The design set specifications for the optics to contain the achromatically tuned beam in the $50 \mathrm{~cm}$-diameter, 1-meter long steel pipe all along the beam line up until the entrance of the first dipole, then to the second dipole where it gets bent and dispersed for momentum analysis. The spectrometer can handle electron beams of energies up to $550 \mathrm{MeV}$ through its quadrupole and dipole magnets that can sustain fields up to $3.5 \mathrm{kGm}$ and $10 \mathrm{kG}$, respectively. $\mathrm{BeO}$ detectors will be installed on insertable plungers for intermediate beam position monitoring, and a scintillating fiber detector is used to detect the electron beam exiting the spectrometer (Fig. 5). The momentum acceptance of this magnetic system is about $40 \%$ in average (Fig. 6). In the early stages of setting up the spectrometer, as is the case next section, a Kodak LANEX scintillating screen was used to image the electron beam, and only the first QQQD part of the spectrometer was used. To investigate the effect of the background (secondary) particles generated when the electron beam hits the pipe, a GEANT simulation was developed.

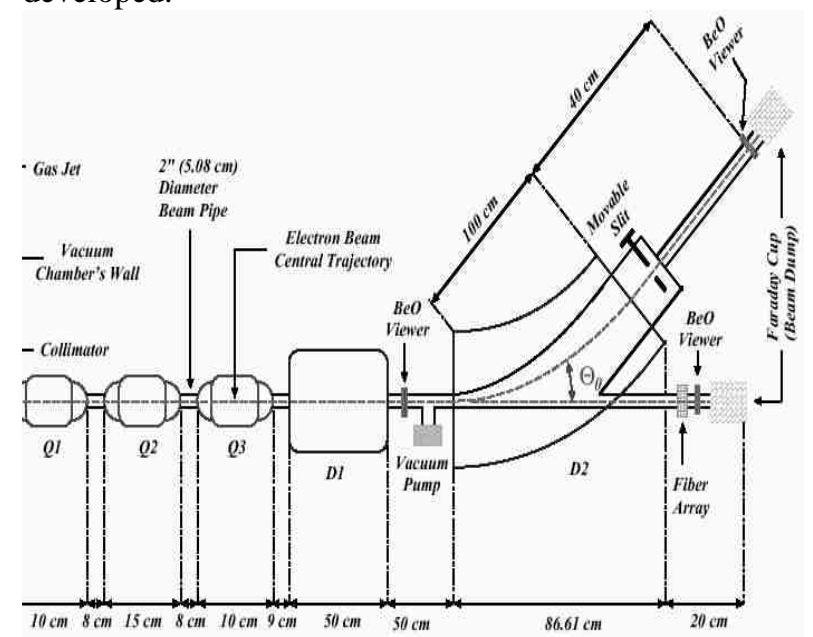

Fig 5: QQQDD spectrometer layout 


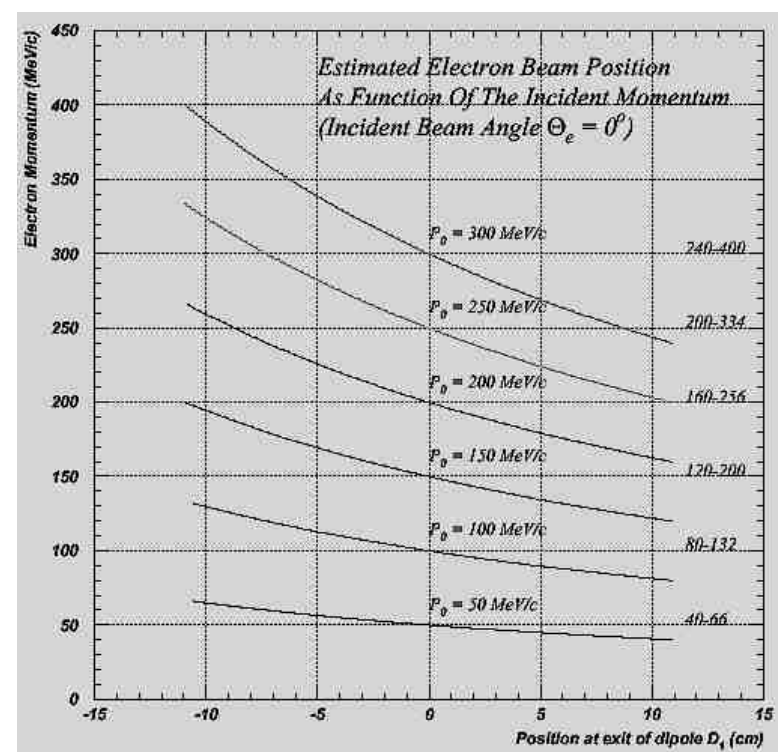

Fig 6: Dispersion curve for the QQQDD spectrometer

\subsection{Implementation and Preliminary Data}

The QQQD spectrometer described above has been constructed and connected to the experimental chamber where the electrons are generated. The fields of the magnets were mapped at the MageTest facility in Jefferson National Laboratory. Fig. 7 depicts the preliminary data collected by a CCD camera capturing the scintillation light from the LANEX screen mounted on the interior of a Plexiglas window at the downstream end of the spectrometer. From the magnetic field values and beam displacement, the momentum of the recorded electrons spans up to about $50 \mathrm{MeV} / \mathrm{c}$.

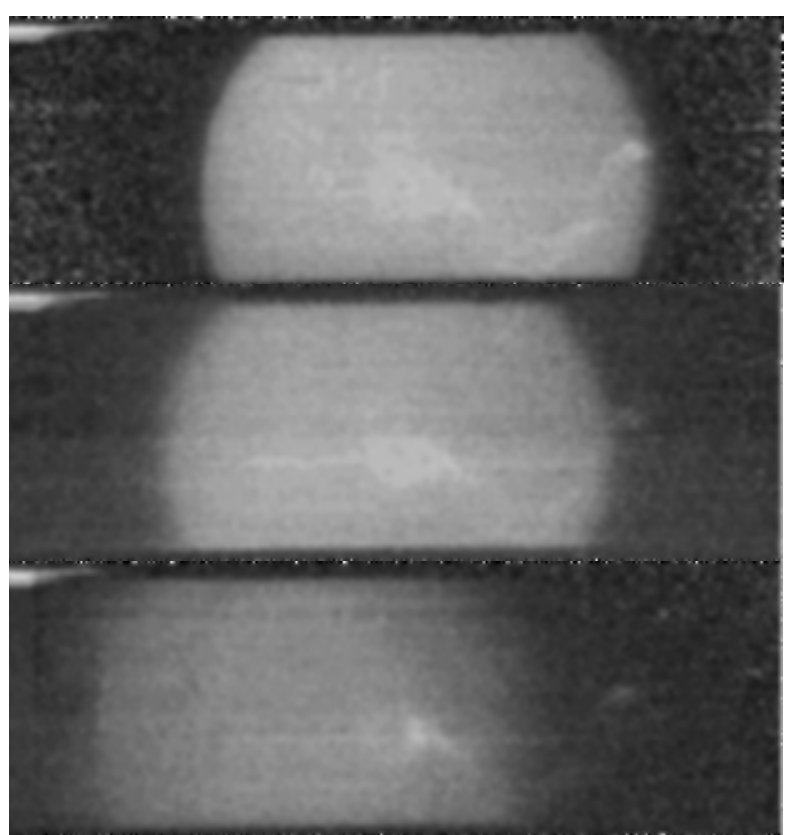

Fig 7: Electron beam drift at the end of the spectrometer as it hits the LANEX screen, the magnetic field increases for the lower pictures.

\section{REFERENCES}

[1] D. Umstadter et al., Phys. Rev. Let., vol. 76, 2073 (1996).

[2] S-Y. Chen et al., Phys. Plasma., vol. 6, 4739 (1999).

[3] See invited talk by D. Umstadter in this proceedings for more details.

[4] X.Wang et al., Phys. Rev. Let., vol. 84, 5324(2000).

[5] N. Saleh et al., Adv. Acc. Conc., AIP conf. Proc., vol. 569, 127 (2000).

[6] N. Saleh et al., Rev. Sci. Instrm., vol. 71, 2305 (2000).

[7] F. Amiranoff et al., NIM-A, vol. 363, 497 (1995). Also, a similar report from Rutherford Appleton Laboratories by Clayton et al., in 1996. 